\title{
The Evaluation of Nutritional Status of 100 Consecutive Patients at the Time of Admission to Surgical Clinic of Oncology Hospital
}

\section{Onkoloji Hastanesi Genel Cerrahi Kliğine Yatan 100 Ardışık Hastanın Yatış Anındaki Nütrisyonel Durumunun Değerlendirilmesi}

\author{
Niyazi Karaman ${ }^{1}$, Osman Uyar ${ }^{3}$, Bahadır Öndeș ${ }^{1}$, Bülent Aksel ${ }^{1}$, Volkan Kınaş ${ }^{2}$, Cihangir Özaslan ${ }^{1}$ \\ ${ }^{1}$ S.B Ankara Onkoloji EA Hastanesi, Genel Cerrahi Kliniği, Ankara, Türkiye \\ ${ }^{2}$ S.B Samsun EA Hastanesi, Genel Cerrahi Kliniği, Samsun, Türkiye \\ ${ }^{3}$ S.B Ceylanpınar Devlet Hastanesi, Genel Cerrahi Kliniği, Urfa, Türkiye
}

Dergiye Ulaşma Tarihi:21/07/2015 Dergiye Kabul Tarihi:11/08/2015 Doi: 10.5505/aot.2015.57060

\section{ÖZET:}

GİRIŞ ve AMAÇ: Bu çalışmada tersiyer bir referans merkezi olan Onkoloji Hastanesi genel cerrahi kliniğinde malignite tanısı ile yatarak tedavi edilmiş 100 ardışık hastanın yatış anındaki nütrisyonel durumunun değerlendirilmesi amaçlanmıştır. YÖNTEM ve GEREÇLER: Hastaların tanıları, cinsiyetleri, vücut kitle indeksleri (VKİ), nütrisyonel risk taraması (NRS 2002) ve nütrisyon durumunun subjektif global değerlendirmesi (SGD) yapılmıştır. Nütrisyon durumunda bozulma açısından hastalar normal (skor 0), hafif bozulma(skor 1), orta bozulma (skor 2) ve şiddetli bozulma (skor 3) olarak gruplandırılmıștır. VKİ açısından; <20 zayıf, 20-25 arası normal kilolu, 25-30 arası kilolu, 30-40 arası şişman ve $>40$ morbid obez olarak değerlendirilmiştir. SGD açısından; A iyi beslenmiş, B hafif-orta beslenme bozukluğu ve C ise şiddetli beslenme bozukluğu olarak değerlendirilmiştir.

BULGULAR: VKİ açısından değerlendirildiğinde; 46 hasta normal kilolu, 34 hasta kilolu, 12 hasta şişman, 7 hasta zayıf ve bir hasta da morbid obez olarak saptanmıştır. Nütrisyon durumunda bozulma açısından değerlendirildiğinde; hastaların 42'si normal bulunurken, 40'ında şiddetli bozulma, 12'sinde orta bozulma ve 6'sında hafif bozulma saptanmıştır. SGD açısından değerlendirildiğinde; 50 hasta iyi beslenmiş olarak değerlendirilirken, 40 hastada hafif-orta derecede beslenme bozukluğu ve 10 hastada ise şiddetli beslenme bozukluğu saptanmıştır. Hastalar tanı sıklığına göre değerlendirildiğinde; 34 hastanın meme kanseri, 24 hastanın mide kanseri, 16 hastanın kolon kanseri ve 8 hastanın da rektum kanseri tanısı ile kliniğe yatırıldıkları saptanmıştır.

TARTIŞMA ve SONUÇ: Onkoloji kliniklerine yatan hastalarda beslenme bozuklukları sanılandan daha sıktır. Bu bozulma hastalığın primer olarak yerleştiği organa göre de değişiklik göstermektedir. Bu hastaların tümü yatış sırasında beslenme durumu açısından değerlendirilmeli ve gelişmiş olan bozukluklar tedavi edilmeli ve gelişebilecek yeni sorunlar engellenmelidir.

Anahtar Kelimeler: Nütrisyonel risk değerlendirme, Onkoloji, Subjektif global değerlendirme, Vücut kitle indeksi

ABSTRACT
INTRODUCTION: Advances in non-small lung cancer (NSCLC) treatment is limited. Disease progression is seen in most patients after first line chemotherapy and many patients receive second line chemotherapy. A prospective study is planned to compare the life quality of NSCLC patients who received second line chemotherapy and those without second line chemotherapy.

METHODS: EORTC QLQ-C30 Version 3.0 (Turkish version) is used to evaluate the quality of life of the patients. The questionnaire is given to the patients at baseline and repeated two times with monthly intervals.

RESULTS: Twenty-four of the total 40 patients entered the study received second line chemotherapy and 16 patients received only supportive care. The general health status, overall function, overall symptom, physical function, role function, emotional function, social function, fatigue symptoms and pain symptoms scores were significantly better in patients who received second line chemotherapy. Cognitive function and dyspnea symptom scores were significantly better after second month in the patients who received second line chemotherapy. There were no statistical differences for nausea, vomiting, anorexia, constipation, diarrhea symptom scores between groups during the first and second month. While insomnia and financial difficulty symptoms scores were significantly better at first month in patients who received second line chemotherapy; no difference is seen in second month.

DISCUSSION AND CONCLUSION: Our findings shows that second line chemotherapy in advanced NSCLC patients significantly improve the general quality of life when compared to only supportive care.

Keywords: Nutritional risk evaluation, oncology, subjective global assessment, body mass index

\section{Giriş}

Malnütrisyon, yetersiz beslenme veya hastalıklarla ilişkili olarak alınan besinlerin emiliminde ve/veya kullanımındaki bozukluklara bağlı olarak gelișen bir durumdur. Hill ve arkadaşlarının yaptığı bir çalışmada medikal hastaların \%40'1nda, cerrahi hastaların $\% 50$ 'sinde malnütrisyon tespit edilmiş ve 
hastanede yatış arttıkça bu oranların daha da arttığı saptanmıştır (1). Cerrahi hastalarda malnütrisyon oranları \%22-54 arasında değişkenlik göstermekte olup, bu değişkenlik yaş, cinsiyet, mevcut hastalık gibi parametrelere bağlıdır (2). Malnütrisyonun; kas fonksiyonları, solunum fonksiyonları ve immün sistem fonksiyonlarında azalmaya, yaşam kalitesi ve yara iyileşmesinde bozulmaya neden olduğu bilinmektedir (3-6).

Cerrahi hastalarda malnütrisyon ciddi bir klinik problemdir ve hastanede yatış süresinin uzaması, artmış mortalite ve morbidite oranları ile ilişkilidir (7-8).

Vücut kitle indeksi (VKİ), vücut ağırlığının (kg), boy uzunluğunun metre cinsinden karesine bölünmesiyle hesaplanır. Yaş ilerledikçe vücut kitle indeksinde artış olabilir. İdeal ağılık ise ulaşılmak istenen VKİnin, boy uzunluğunun karesi ile çarpılmasıyla elde edilir.

Günümüzde en yaygın kullanılan tarama yöntemi NRS 2002'dir. ESPEN (European society of Parenteral and Enteral Nutrition) nütrisyon değerlendirmesinde NRS 2002 yöntemini önermektedir. NRS 2002 de hastalar beslenme yetersizliği ve hastalık şiddeti bakımından değerlendirilir ve şu şekilde skorlanırlar: yok (0), hafif (1), orta şiddette (2) ve ağır (3). Toplam skoru $\geq 3$ olan hastalar beslenme riski altında olarak değerlendirilirler.

Subjektif Global Değerlendirme (SGD) de çok yararlı ve sık uygulanan bir testtir. Ancak uygulamada subjektivite içermektedir. Fonksiyonel kapasite ve malnütrisyonda gözlenen fizyolojik belirtiler veya malnütrisyonun içerdiği durumları değerlendirmek amaciyla geliştirilmiştir. Hastanın hikayesi (ağırlık kaybı, besin alımında değişme, gastrointestinal semptomlar ve fonksiyonel durum), fiziksel testler (kas kitlesi, subkutan yağ, sakral ve ayak ödemi, asit) gibi değerlendirme kriterlerini içermektedir. SGA laboratuar verilerini içermez.

$\mathrm{Bu}$ çalışmada tersiyer bir referans merkezi olan Onkoloji Hastanesi genel cerrahi kliniğinde malignite tanısı ile yatarak tedavi edilmiş 100 ardışık hastanın yatış anındaki nütrisyonel durumunun değerlendirilmesi amaçlanmıştır.

\section{Gereç ve yöntem}

Genel Cerrahi kliniğinde yatarak tedavi edilmiş 100 ardışık hastanın tanıları, cinsiyetleri, vücut kitle indeksleri (VKİ), nütrisyonel risk taramas1 (NRS 2002) ve nütrisyon durumunun subjektif global değerlendirmesi (SGD) yapılmıştır. Nütrisyon durumunda bozulma açısından hastalar normal (skor 0), hafif bozulma (skor 1), orta bozulma (skor 2) ve şiddetli bozulma (skor 3) olarak gruplandırılmıştır. VKİ açısından; $<20$ zayıf, 20-25 aras1 normal kilolu, 25-30 arasi kilolu, 30-40 arası şişman ve $>40$ morbid obez olarak değerlendirilmiștir. SGD açısından; A iyi beslenmiş, B hafif-orta beslenme bozukluğu ve $\mathrm{C}$ ise şiddetli beslenme bozukluğu olarak değerlendirilmiştir.

\section{Sonuçlar}

Genel cerrahi kliniğine yatış için kabul edilen hastaların cinsiyet dağılımı 60 kadın, 40 erkek şeklindedir. VKİ açısından değerlendirildiğinde; 46 hasta normal kilolu, 34 hasta kilolu, 12 hasta şişman, 7 hasta zayıf ve bir hasta da morbid obez olarak saptanmıştır. Nütrisyon durumunda bozulma açısından değerlendirildiğinde; hastaların 42'si normal bulunurken, 40'inda şiddetli bozulma, 12'sinde orta bozulma ve 6'sinda hafif bozulma saptanmıştır. SGD açısından değerlendirildiğinde; 50 hasta iyi beslenmiş olarak değerlendirilirken, 40 hastada hafif-orta derecede beslenme bozukluğu ve 10 hastada ise şiddetli beslenme bozukluğu saptanmıştır (Tablo 1).

Hastalar tanı siklığına göre değerlendirildiğinde; 34 hastanın meme kanseri, 24 hastanın mide kanseri, 16 hastanın kolon kanseri ve 8 hastanın da rektum kanseri tanısı ile kliniğe yatırıldıkları saptanmıştır. Meme kanseri tanılı hastalar VKİ açısından değerlendirildiğinde; 16 hasta normal kilolu, 11 hasta kilolu, 6 hasta şişman, 7 ve bir hasta da morbid obez olarak saptanmıştır. Nütrisyon durumunda bozulma açısından değerlendirildiğinde; hastaların 27'si normal bulunurken, 3 'er hastada orta ve hafif bozulma ve bir hastada şiddetli bozulma saptanmıştır. SGD açısından değerlendirildiğinde; 30 hasta iyi beslenmiş olarak değerlendirilirken, 4 hastada hafif-orta derecede beslenme bozukluğu saptanmıştır.

Mide kanseri tanılı hastalar VKİ açısından değerlendirildiğinde; 12 hasta 
normal kilolu, 6 hasta kilolu, 4 hasta da zayıf ve olarak saptanmıştır. Nütrisyon durumunda bozulma açısından; hastaların 16'sında şiddetli bozulma saptanırken, 3 hastada orta ve bir hastada da hafif bozulma saptanmıştır. SGD açısından; 15 hastada hafif-orta derecede ve 5 hastada şiddetli beslenme bozukluğu saptanmıştır.

Kolon kanseri tanıl hastalar VKİ açısından değerlendirildiğinde; 9 hasta kilolu, 5 hasta normal kilolu, 2 hasta da şişman olarak saptanmıştır. Nütrisyon durumunda bozulma açısından; hastaların 9'unda şiddetli bozulma saptanırken, 4 hastada orta derecede bozulma saptanmıştır. SGD açısından; 9 hastada hafiforta derecede ve 2 hastada şiddetli beslenme bozukluğu saptanmıştır.

Rektum kanseri tan1lı hastalar VKİ açısından değerlendirildiğinde; 4 hasta kilolu, 3 hasta normal kilolu ve 1 hasta da zayıf olarak saptanmıştır. Nütrisyon durumunda bozulma açısından; hastaların 4'ünde (\%50) şiddetli bozulma saptanırken, 1 hastada orta derecede bozulma saptanmıştır. SGD açısından; 6 hastada hafif-orta derecede beslenme bozukluğu saptanmıştır.

\begin{tabular}{|c|c|c|c|c|c|}
\hline & $\begin{array}{l}\text { GENEL } \\
\text { n }(\%)\end{array}$ & $\begin{array}{c}\text { MEME CA } \\
\text { n (\%) }\end{array}$ & $\begin{array}{c}\text { MIDEE CA } \\
\text { n (\%) }\end{array}$ & $\begin{array}{c}\text { KOLON CA } \\
\text { n(\%) }\end{array}$ & $\begin{array}{c}\text { REKTUM CA } \\
\text { n (\%) }\end{array}$ \\
\hline \multicolumn{6}{|l|}{ BMI } \\
\hline$<20$ & $7(7)$ & 0 & 4 (16.6) & 0 & $1(12.5)$ \\
\hline $20-25$ & $46(46)$ & $16(47.0)$ & $12(50)$ & $5(31.2)$ & $3(37.5)$ \\
\hline $25-30$ & $34(34)$ & $11(32.3)$ & $6(25)$ & $9(56.2)$ & $4(50)$ \\
\hline $30-40$ & $12(12)$ & 6 (17.6) & $2(8.33)$ & $2(12.5)$ & 0 \\
\hline$>40$ & $1(1)$ & $1(2.94)$ & 0 & 0 & 0 \\
\hline NRS & -- & -- & -- & -- & -- \\
\hline SKOR 0 & $42(42)$ & $27(79.4)$ & $4(16.6)$ & $3(18.7)$ & $3(37.5)$ \\
\hline SKOR 1 & $6(6)$ & $3(8.82)$ & $1(4.16)$ & 0 & 0 \\
\hline SKOR 2 & $12(12)$ & $3(8.82)$ & $3(12.5)$ & $4(25)$ & $1(12.5)$ \\
\hline SKOR 3 & $40(40)$ & $1(2.94)$ & $16(66.6)$ & $9(56.2)$ & $4(50)$ \\
\hline SGD & -- & -- & -- & -- & -- \\
\hline A & $50(50)$ & $30(88.2)$ & $4(16.6)$ & $5(31.2)$ & $2(25)$ \\
\hline $\mathrm{B}$ & $40(40)$ & $4(11.7)$ & $15(62.5)$ & $9(56.2)$ & $6(75)$ \\
\hline $\mathrm{C}$ & $10(10)$ & 0 & $5(20.8)$ & $2(12.5)$ & 0 \\
\hline TOPLAM & 100 & 34 & 24 & 16 & 8 \\
\hline
\end{tabular}

Tablo1. Hastaların beslenme durumlarının tanılarına göre değerlendirilmesi

\section{Tartışma}

Pek çok ciddi araştırmanın sonuçlarına göre, Avrupa'da, sağlıklı halkın \%5-15'i, hastaneye yatan hastaların $\% 40$ ' 1 ve bakım evinde yaşayanların \%60'1 malnütrisyonda veya nütrisyon riski altındadır $(9,10,11)$. Klinik Enteral Parenteral Nütrisyon Derneği, ülkemizdeki durumu görmek üzere 2005 2006 y1llarında 19 şehirde ve 34 merkezde 6 ay süren bir araştırma yapmış, NRS-2002 tarama yöntemi kullanılmış ve yaklaşık 35 bin hasta değerlendirilmiştir (12). Bu çalışmada, yatış anında hastaların ortalama \%15'inin nütrisyonel açıdan risk altında olduğu gösterilmiştir. Bizim çalışmamızda malnütrisyon oranlarının daha yüksek olmasının nedeni değerlendirilen hastaların malignite tanısı almış olmalarıdır. Çünkü yapılan bir çalışmada malignitenin malnütrisyon için bağımsız risk faktörü olduğu belirtilmiştir (13). Bu yüzden hastaneye yatan hastalarda nütrisyon durumunun yatış anında değerlendirilmesi önemlidir. Özellikle gastrointestinal maligniteye bağlı olarak cerrahi planlanan hastalarda nütrisyon durumu yapılacak cerrahinin sonuçlarında anahtar bir role sahiptir (14). NRS ve SGD değerleri malnütrisyon ve majör abdominal cerrahi komplikasyonları açısından prediktif olabilir (14). $\mathrm{Bu}$ yüzden nütrisyon durumunun değerlendirilmesi ve tedavi edilmesi, postoperatif morbidite ve mortalite oranlarının azalmasını, hastanede yatış süresinin kısalmasını, yaşam kalitesinin artmasını ve maliyet oranlarının azalmasını sağlayabilir.

Hastaneye yatan hastalar arasinda nütrisyonel risk ve malnütrisyon oldukça s1k karşılaşılan bir durumdur. $\mathrm{Bu}$ nedenle çok değişik nütrisyonel tanı yöntemleri tanımlanmıştır. Bunlar arasında karşılaştırmalı 
değerlendirmenin yapıldığ 2002'nin ve biyoelektrik impedans analizinin parametrelerinin bozulmuş nütrisyonel durumu saptamada daha üstün oldukları bildirilmiştir (15). Kritik hastalıklarda nütrisyon açısından riskli hastaların saptanması zordur. Subjektif olmaları nedeniyle geleneksel tarama ve değerlendirme yöntemlerinin kullanımı sinırlıdır. Nütrisyonel riskin değerlendirilmesinde fiziksel değerlendirme, fonksiyonel durum ve hastalığın ciddiyeti gibi parametrelerin de hesaba katılması daha uygun olabilir (16). Hastaneye yatan hastalarda malnütrisyon varlığı komplikasyon ve ölüm oranlarının artmasına neden olur. SGD'nin, diğer nütrisyon tarama yöntemleri ile karşılaştırıldığında, kritik düzeyde hastalığ1 olmayan hastalarda klinik olarak etkin ve basit bir yöntem olduğu ve hastane mortalite riskini diğer indekslerden daha iyi belirlediği bildirilmiştir (17). Mide ve kolorektal kanserli hastaların nütrisyonel durumlarının hastane dışında değişik metodlar ile değerlendirildiği bir çalışmada hasta kaynaklı SGD'nin diğer objektif metodlardan daha hassas olduğu sonucuna varılmış ve kanser hastalarında tedavi öncesi ve sonrası universal malnutrisyon taramasi (MUST) ile birlikte kullanıması önerilmiştir (18).

Sonuç olarak onkoloji kliniklerine yatan hastalarda beslenme bozuklukları sanılandan daha sıktır. Bu bozulma hastalığın primer olarak yerleştiği organa göre de değişiklik göstermektedir. Bu hastaların tümü yatış sırasında beslenme durumu açısından değerlendirilmeli ve gelişmiş olan bozukluklar tedavi edilmeli ve gelişebilecek yeni sorunlar engellenmelidir.

\section{Çıkar Çatışması: Yok}

\section{Kaynaklar:}

1. Hill GL, Blackett RL, Pickford I, Burkinshaw L, Young GA, Warren JV, et al. Malnutrition in surgical patients. An unrecognised problem. Lancet 1977; 1: 689-692

2. Bruun LI, Bosaeus I, Bergstad I, Nygaard K. Prevalence of malnutrition in surgical patients: evaluation of nutritional support and documentation. Clin Nutr 1999; 18: 141-147
3. Arora NS, Rochester DF. Respiratory muscle strength and maximal voluntary ventilation in undernourished patients. Am Rev Respir Dis 1982; 126: 5-8

4. Chandra RK. Nutrition, immunity, and infection: present knowledge and future directions. Lancet. 1983;1: 688-691

5. Larsson J, Akerlind I, Permerth J, Hörnqvist JO. The relation between nutritional state and quality of life in surgical patients. Eur J Surg 1994; 160: 329-334

6. Haydock DA, Hill GL. Improved wound healing response in surgical patients receiving intravenous nutrition. Br J Surg 1987; 74: 320-323

7. Robinson G, Goldstein M, Levine GM. Impact of nutritional status on DRG length of stay. JPEN J Parenter Enteral Nutr 1987; 11: 49-51

8. Reilly JJ Jr, Hull SF, Albert N, Waller A, Bringardener S. Economic impact of malnutrition: a model system for hospitalized patients. JPEN J Parenter Enteral Nutr 1988; 12: 371-376

9. McWhirter JP, Pennington CR. Incidence and recognition of malnutrition in hospital. BMJ 1994; 308: 945-948

10. Pirlich M, Schütz T, Norman K, Gastell S, Lübke HJ, Bischoff SC, et al. The German hospital malnutrition study. Clin Nutr 2006; 25: 563-572

11. Elia M, Jones B, Russell C. Malnutrition in various care settings in the UK: the 2007 Nutrition Screening Week Survey Clin Med 2008; 8: 364-365

12. Korfali G, Gündoğdu H, Aydintuğ S, Bahar M, Besler T, Moral AR, et al. Nutritional risk of hospitalized patients in Turkey. Clin Nutr 2009; 28 : 533-537

13. Waitzberg DL, Caiaffa WT, Correia MI. Hospital malnutrition: the Brazilian national survey (IBRANUTRI): a study of 4000 patients. Nutrition 2001; 17: 573-580

14. Hülya Sungurtekin, Uğur Sungurtekin, Canan Balci, Mehmet Zencir and Ergün Erdem. The influence of nutritional status on complications after major intraabdominal surgery. Journal of the American College of Nutrition 2004; 23: 227-232

15. Meireles MS, Wazlawik E, Bastos JL, Garcia MF. Comparison between nutritional risk tools and parameters derived from bioelectrical impedance analysis with subjective global assessment. J Acad Nutr Diet. 2012; 112: 1543-1549

16. Coltman A, Peterson S, Roehl K, Roosevelt H, Sowa D. Use of 3 tools to assess nutrition risk in the intensive care unit. JPEN J Parenter Enteral Nutr. 2015; 39: 28-33

17. Olveira G, Tapia MJ, Ocón J, Cabrejas-Gómez C, Ballesteros-Pomar MD, Vidal-Casariego A, et al. The subjective global assessment predicts in-hospital mortality better than other nutrition-related risk indexes in noncritically ill inpatients who receive total parenteral nutrition in Spain (prospective multicenter study). J Acad Nutr Diet. 2013; 113: 1209-1218

18. Abe Vicente M, Barão K, Silva TD, Forones NM. What are the most effective methods for assessment of nutritional status in outpatients with gastric and colorectal cancer? Nutr Hosp. 2013; 28: 585-591 